\title{
Plasmon Mediated Photocatalysis by Solar Active Ag/ZnO Nanostructures: Degradation of Organic Pollutants in Aqueous Conditions
}

\author{
Aby $\mathrm{H}$, Kshirsagar A and Khanna $\mathrm{PK}^{*}$ \\ Department of Applied Chemistry, Defence Institute of Advanced Technology (DU), DRDO, Govt. of India \\ Girinagar, Pune 411025, India
}

*Corresponding author: Khanna PK, Department of Applied Chemistry, Defence Institute of Advanced Technology (DU), DRDO, Govt. of India Girinagar, Pune 411025, India, Email: pawankhanna2002@yahoo. co.in

Citation: Aby H, Kshirsagar A, Khanna PK (2016) Plasmon Mediated Photocatalysis by Solar Active Ag/ ZnO Nanostructures: Degradation of Organic Pollutants in Aqueous Conditions. J Mater Sci Nanotechnol 4(1): 103. doi: 10.15744/2348-9812.4.103

Received Date: October 01, 2015 Accepted Date: January 26, 2016 Published Date: January 28, 2016

\begin{abstract}
Present article focuses on the in-situ generated $\mathrm{Ag} / \mathrm{ZnO}$ nano catalyst for effluent treatment. It is observed that synthesis of the catalyst using starch as environment friendly surfactant resulted in plate like elongated Zinc Oxide nanoparticles. As-synthesized catalyst is characterized by X-Ray Diffraction measurement confirming hexagonal $\mathrm{ZnO}$ and fcc silver. XRD, further revealed d-spacings of 0.26 and $0.234 \mathrm{~nm}$ for $\mathrm{ZnO}$ and $\mathrm{Ag} / \mathrm{ZnO}$ indicating the correctness of their crystal structures. FESEM, EDX and TEM, showed spherical but flat plate type morphology due to presence of starch. XPS confirmed oxygen rich composition and UV-Vis spectrophotometry revealed decrease in band gap energies with increasing silver loading. The photocatalytic performance of the plasmonic catalysts was studied under direct sunlight. Degradation of aqueous solutions of Methylene Blue (MB) Rhodamine B (RhB), Methyl Orange (MO), Bromophenol Blue (BPB) and p-nitrophenol (PNP) was monitored by absorption spectroscopy to reveal complete degradation of organic dyes. It was observed that the catalyst retained its properties even after third recycle. Such simple catalyst may offer ready solution for effluent treatment under direct sunlight.
\end{abstract}

Keywords: Plasmonic Photocatalysis; Semiconductor; Advanced Oxidation Process; Dye Degradation

List of abbreviations: MB: Methylene Blue; RhB: Rhodamine B; MO: Methyl Orange; BPB: Bromophenol Blue; PNP: p-nitrophenol

\section{Introduction}

Photocatalytic property of semiconductor materials has been a topic of extensive research, ever since splitting of water with $\mathrm{TiO}_{2}$ was achieved under irradiation of light by Fukushima and Honda in 1971 [1]. Among the semiconductor materials suitable for photocatalytic reactions, $\mathrm{TiO}_{2}$ and $\mathrm{ZnO}$ received much attention due to their easy availability, non toxicity, thermal and chemical stability [2-4] and varied applications such as photo-degradation of the organic pollutants [5,6], artificial photosynthesis [7] and splitting of water [8]. It has been documented that $\mathrm{ZnO}$ is more efficient than $\mathrm{TiO}_{2}$ for degradation of water pollutants [9] despite their similar band gap energy (i.e. $\sim 3.2 \mathrm{eV}$ ). Lower electric potentials of the valence and conduction band, $\mathrm{ZnO}(-0.45$ to $2.75 \mathrm{eV})$ when compared to $\mathrm{TiO}_{2}(-0.1$ to $3.1 \mathrm{eV})$ enables $\mathrm{ZnO}$ to effectively perform photocatalytic degradation reactions at lower potentials $[10,11]$. Additionally, higher quantum efficiency is an added advantage for $\mathrm{ZnO}_{\text {over }} \mathrm{TiO}_{2}$ as a photocatalyst for degradation of water pollutants [12].

Photocatalytic degradation of organic pollutants is environment friendly as during the process, the organic pollutants are converted to $\mathrm{CO}_{2}$ and $\mathrm{H}_{2} \mathrm{O}$ without generating any harmful by-products $[13,14]$. Often the laboratory photocatalytic reactions are performed using simulated sunlight produced via xenon lamps and actual performance of the catalyst is not tested in real sunlight. In order to make the process more attractive, it is desirable to conduct such degradation experiments under direct sunlight, $50 \%$ of which is constituted by ultraviolet-visible radiation. $\mathrm{ZnO}$ by virtue of its band-gap limits its activity to UV region (3-4\% of sunlight). To make $\mathrm{ZnO}$ as an effective catalyst in the Visible region ( $44 \%$ of sunlight), its optical property is required to be tuned in the visible range i.e. between $400 \mathrm{~nm}$ to $700 \mathrm{~nm}$ [15]. To alter the band-gap of $\mathrm{ZnO}$ numerous techniques like creating structural defects [16], synthesizing particles of various morphology, doping etc are adopted. Similarly, morphology of the materials can play effective role in light harvesting thus various synthesis routes have been explored to improve the crystalline quality of nanoparticles [16-21] of varying shapes and sizes like nanorods [18], nanoflowers 19], nanowires [20], and nanosheets [21] for their utility as visible light photocatalysts. 
Among surfactants employed to control the particle shape and size, bio-polymers like starch are considered as an effective and environment friendly option. It has been reported that polymeric nature of starch acts as a template for the formation of highly crystalline nanoparticles as it is a green precursor and offers economically viable option for production of $\mathrm{ZnO}$ [22-25].

According to the literature reports, $\mathrm{TiO}_{2}$ has been extensively used as one of the most effective semiconductor photocatalysts. A quick recombination of charge carriers upon exposure to light is the major limitation to achieve high photocatalytic efficiency against pollutants. Recombination of electrons and hole pairs has faster kinetics and therefore, it reduces the quantum efficiency of photocatalysis. In order to promote the photocatalysis efficiency it is very important to decrease the recombination rate. This photocorrosion problem can be solved by surface modification of photocatalyst which may change the charge, functionality, reactivity of the surface and enhance stability. There are several reports describing the prohibition of the photocorrosion by surface modification some of the important examples are discussed here. Photocorrosion suppression was achieved by Zhu et al via hybridization of $\mathrm{ZnO}$ with graphite like carbon [25]. Another report on the photoelectrochemical water splitting using carbon and nitrogen co-treated $\mathrm{ZnO}$ nanorod arrays describes the enhancement in the catalytic activity with decreased photocorrosion due to surface modification. The enhancement in the photocatalytic performance and suppression of photocorrosion of $\mathrm{ZnO}$ via coupling with carbon materials and reduced graphene oxide showed good results in the photocatalysis [26].

Doping of $\mathrm{ZnO}$ [26] with metals [27], non metals [28], other compounds [29] and semiconductor materials [15] can also be considered to enhance visible range photocatalytic activity. Among them, doping with a noble metal (Ag and Au) is the most sought after idea due to LSPR (a unique property of conduction band electrons of noble metals to oscillate by incident light). Noble metal doping also limits recombination of electron hole pairs [30] due to the formation of Schottky junction and such noble metal doped catalysts are sometime referred to as plasmonic photocatalysts thus silver has attracted much attention as dopant [31,32]. Ag doped $\mathrm{ZnO}$ has been studied by several researchers e.g. Georgekutty [33] et al. reported the visible light performance of $\mathrm{Ag}$ doped $\mathrm{ZnO}$ particles by monitoring the degradation reaction of Rhodamin 6G (R6G). Mondal [34] et al. studied the direct sunlight performance of $\mathrm{ZnO}$ photocatalysts by doping it with Au by monitoring a spectrum of dyes, but efficiency of degradation was not attractive. Zhang [22] et al. synthesized highly crystalline micro porous ZnO using starch as surfactant showing excellent catalytic properties under UV radiation. The studies demonstrated that, high oxygen content in semiconductor can improve the visible light photocatalytic properties of semiconductor [2]. Though one pot synthesis of the Ag/ $\mathrm{ZnO}$ is reported, still precipitation of Ag nanoparticles on the surface can be considered as an excellent option since it will facilitate control over the amount of silver particles. The authors have earlier demonstrated synthesis and applications of $\mathrm{ZnO}, \mathrm{TiO}_{2}$ and silver nano-particles and herewith extending their efforts in photocatalysis [22].

The present study relates with the idea of designing a highly solar active silver doped ZnO photocatalyst using starch and its sintering at a lower temperature to retain oxygen rich surfaces. In order to study the photocatalytic performance, Methylene Blue was chosen as the primary dye. Degradation of other organic dyes like Rhodamine B, Methyl Orange, Bromophenol Blue and p-nitrophenol were also monitored. To the best of our knowledge, the present report is probably the first to describe solar degradation of p-nitrophenol using Ag doped $\mathrm{ZnO}$.

\section{Experimental}

\section{Materials}

All reagents used were of analytical grade and were purchased from commercial sources. The water used for the experiment is ultrapure water from Millipore Direct Q 3UV purifier system.

\section{Instrumentation}

Powder X-Ray Diffraction was performed using Bruker A8 advanced with monochromatized CuKa radiation with an accelerating voltage of $40 \mathrm{kV}$ and an applied current of $20 \mathrm{~mA}$. Field Emission Scanning Electron Microscopy (FESEM) and EDAX were performed with a supra 40, Carl Zeiss Pvt. Ltd. Instrument and Oxford link, ISIS 300 to find the nanocrystal composition. Photoluminescence studies were conducted using a Cary Eclipse fluorescence spectrophotometer by Agilent. Transmission Electron Microscope (TEM) images were taken by TECHNAI G2 instrument. X-Ray Photoelectron Spectroscopy (XPS) was done with ESCA-3000 (VG Microtech UK) having Mg Ka and Al Ka source. All UV-Visible spectroscopic analysis were done on Specord 210 plus (Analytik Jena). The sunlight intensity was measured using a Lutron LX-101A light meter. The photocatalytic activities were performed under direct sunlight with average intensity of about 86,500 LUX as measure by LUX meter.

\section{Synthesis of $\mathrm{ZnO}$ nanoparticles}

Zinc Nitrate $30.00 \mathrm{~g}$ is added to $0.5 \%$ starch solution [11] and the mixture was kept under constant stirring for an hour. After complete dissolution of the zinc nitrate, temperature was increased to $60{ }^{\circ} \mathrm{C}$ and $600 \mathrm{ml} \mathrm{NaOH}$ solution in water $(0.2 \mathrm{M})$ was added to the reaction mixture. The mixture was further stirred at same temperature for another $4 \mathrm{hrs}$. The overall mixture was kept for cooling and ageing overnight. The suspension was centrifuged to collect white precipitate which was washed with ethanol and distilled water twice. White powder so-collected was dried in an oven at $80^{\circ} \mathrm{C}$ for $3-5 \mathrm{hrs}$. 


\section{Synthesis of $\mathrm{Ag} / \mathrm{ZnO}$ nano catalyst}

$1.0 \mathrm{~g}$ of $\mathrm{ZnO}$ nanoparticles were taken and dispersed in $100 \mathrm{ml}$ ethanol via sonication process for $30 \mathrm{minutes}$. AgNO $(40 \mathrm{mg}$ ) was suspended in above solution for preparation of $\mathrm{Ag} / \mathrm{ZnO} 1$. The solution was stirred for 3 hours at a temperature of $45^{\circ} \mathrm{C}$ to obtain a brownish yellow precipitate. The precipitate was centrifuged, collected and washed with ethanol and DI water. Similarly, Ag/ZnO 2 was prepared by adding $160 \mathrm{mg}$ of $\mathrm{AgNO}_{3}$ to the solution/suspension containing same amount of $\mathrm{ZnO}$.

\section{Photocatalytic study}

Photocatalytic performance of the $\mathrm{Ag} / \mathrm{ZnO}$ composition was determined by monitoring the degradation of methylene blue dye in sunlight. $0.02 \mathrm{mM}$ aqueous solution of methylene blue was prepared in distilled water. To the methylene blue solution (30 $\mathrm{mL}$ ), bare $\mathrm{ZnO}$ or $\mathrm{Ag} / \mathrm{ZnO}$ catalysts $(30 \mathrm{mg}$ ) were dispersed to prepare the final reaction solution $(1 \mathrm{~g} / \mathrm{L})$ ready for photocatalytic evaluation. The so-generated solutions were kept in dark and stirred well for 20 minute to achieve adsorption equilibrium between MB and the catalyst surfaces. The solution was placed under direct sunlight with occasional stirring. After reaction, every 20 minute, $2 \mathrm{~mL}$ solution was withdrawn and centrifuged to record UV/Vis spectra of the sample. The procedure was repeated with various concentrations of catalysts. Similar experiments were done with Rhodamine B (RhB), Methyl Orange (MO), Bromophenol Blue (BPB) and p-nitrophenol (PNP)

\section{Results and Discussion}

White crystalline nanoparticles of $\mathrm{ZnO}$ is formed as the result of the reaction.(A in Figure 1). During reaction $\mathrm{Zn}$ ions of Zinc Nitrate interact with $\mathrm{OH}$ radicals of starch networks, which is an important component of the system [24]. The zinc oxide formed get converted to $\mathrm{ZnO}$ nanoparticles by heat treatment at $100-200{ }^{\circ} \mathrm{C}$. The nitrate group get decomposed as $\mathrm{NO}_{2}$ and $\mathrm{Oxygen}$.

The typical reaction can be expressed as:

$$
\begin{aligned}
& \left.\mathrm{Zn}\left(\mathrm{NO}_{3}\right)_{2} \cdot 6 \mathrm{H}_{2} \mathrm{O}+2 \mathrm{NaOH} \rightarrow 2 \mathrm{NaNO}_{3} \text { (aq. }\right)+\mathrm{Zn}(\mathrm{OH})_{2}+6 \mathrm{H}_{2} \mathrm{O} \\
& \mathrm{Zn}(\mathrm{OH})_{2} \rightarrow \mathrm{ZnO}+\mathrm{H}_{2} \mathrm{O}
\end{aligned}
$$

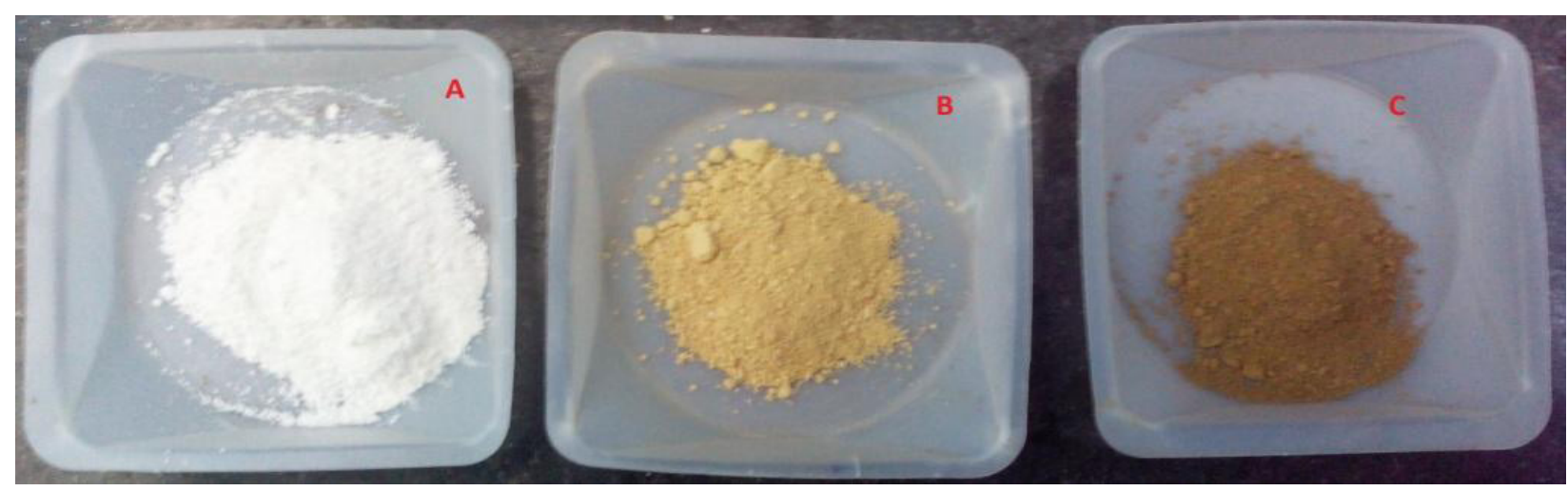

Figure 1: As-synthesized $\mathrm{ZnO}(\mathrm{A}), \mathrm{Ag} / \mathrm{ZnO} 1$ (B) and $\mathrm{Ag} / \mathrm{ZnO} 2$ (C) nanoparticles

Suspension of $\mathrm{ZnO}$ particles capped with starch was made in ethanol and $\mathrm{AgNO}_{3}$ was added to it for loading of silver particles via reduction of silver ions. The process involves reduction of silver salt by the ethanol solution whereby Ag particles are formed on the surface of $\mathrm{ZnO}$. Ethanol is known to be a mild reducing agent and formations of silver nano-particles have been reported by ethanol reduction process by others also [35]. The color changes from white to mild yellow and/or yellow brown for the final silver doped zinc oxide powders (B, C in Figure 1). The typical chemical reaction mechanism could be explained as below;

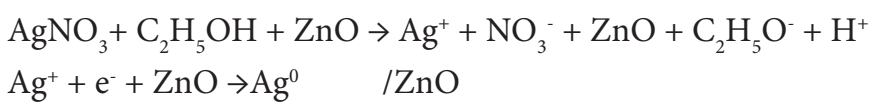

$\mathrm{Ag}^{+}+\mathrm{e}^{-}+\mathrm{ZnO} \rightarrow \mathrm{Ag}^{0} \quad / \mathrm{ZnO}$

The so-generated powders were analyzed by several modern tools e.g. XRD, SEM, TEM, XPS, UV-Vis and PL spectroscopy.

\section{XRD Measurements}

The crystallinity, phase, and purity of the as-prepared samples were determined by powder X-ray diffraction (PXRD). Obtained XRD patterns of the $\mathrm{ZnO}$ and $\mathrm{ZnO} / \mathrm{Ag}$ particles are shown in Figure $2 \mathrm{a}$. For $\mathrm{ZnO}$ alone, ten major peaks are observed at $2 \theta$ values of $31.82,34.47,36.29,47.56,56.53,62.89,66.35,67.96,69.15,72.56$ and $77.46^{\circ}$ which corresponds to the (100), (002), (101), (102), (110), (103), (200), (112), (201), and (202) crystal planes of the hexagonal ZnO structure [22]. The same peaks appear in the XRD pattern of $\mathrm{Ag} / \mathrm{ZnO}$, indicating there are no structural changes due to silver doping however, pattern also shows peaks for fcc Ag. A peak at $2 \theta 38.45^{\circ}$, which is due to the presence of (111) crystal plane of silver particle on $\mathrm{ZnO}$ surface, was an excellent confirmation of formation of silver at $\mathrm{ZnO}$ [18]. The peak at $2 \theta 44.27^{\circ}$ for (200) is also visible but the peak at $2 \theta 64.60^{\circ}$ for (220) crystal planes of fcc metallic Ag are not so clear however indication in the XRD pattern is there was very weak hump. Interestingly, it has been observed that there is a minor red shift in 2 theta values for $\mathrm{ZnO}$ peaks due to presence of silver (Figure $2 \mathrm{~b}$ ), possibly due to intera- 
ction of silver with $\mathrm{ZnO}$ matrix by the formation of Schottky junction [36]. The size of the nanoparticles was estimated by DebyeScherrer equation [22] and was found to be $21.6 \mathrm{~nm}$. The increase in crystalline size of $\mathrm{Ag} / \mathrm{ZnO}$ was only marginal but can be attributed to the addition of $\mathrm{Ag}$ particles to the matrix [37]. The particle size of silver present on the surface or in the matrix of $\mathrm{ZnO}$ was estimated to be $18.9 \mathrm{~nm}$ which suggest that it is in the range to be adsorbed on the surface. The lattice spacing between two planes in $\mathrm{ZnO}$ for 002 plane at $2 \theta$ value of $34.47^{\circ}$ is $0.26 \mathrm{~nm}$. The same is $0.234 \mathrm{~nm}$ for silver present in the Ag/ZnO catalyst for 111 planes of silver at $2 \theta$ value of $38.45^{\circ}$. Lattice spacings for both materials match well with the reported values thereby confirming excellent quality and actual crystal planes and structure of the nano-particles [17]. The presence of oxygen vacancies in the crystal structure of the $\mathrm{ZnO}$ plays very important role in deciding catalytic activity. The oxygen vacancies in present case were confirmed from literature reports [37].
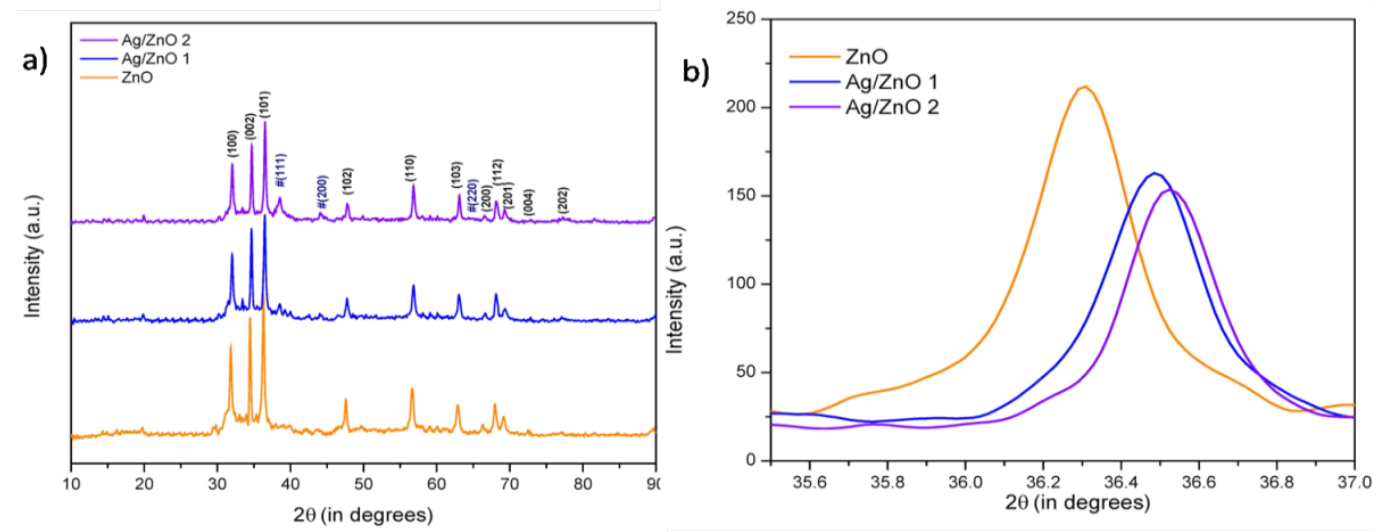

Figure 2: XRD patterns of various samples (a) $\mathrm{ZnO}$ and $\mathrm{Ag} / \mathrm{ZnO}$ (b) 101 plane of same.

\section{SEM/EDAX Analysis}

To study the morphology of $\mathrm{ZnO}$ particles and distribution of Ag particles on the surface of the matrix, SEM analysis was carried out (Figure 3). In the SEM image, the morphology of the matrix $(\mathrm{ZnO})$ is observed to be plate like however flaky in nature but closer to tubular shaped. Such mixed elongated morphology has been described to be useful for energy capturing process [38]. The $\mathrm{Ag}$ doped $\mathrm{ZnO}$ showed spherical particles on the surface of the plate like flakes which no longer retains tubular appearance but are elongated to retain mixed morphology of the powder. The surface of the $\mathrm{ZnO}$ nanoparticles can be seen with uniformly dispersed Ag nanoparticles all over them. The Ag particles are more visible in the sample with larger concentration than the one with lower concentration.
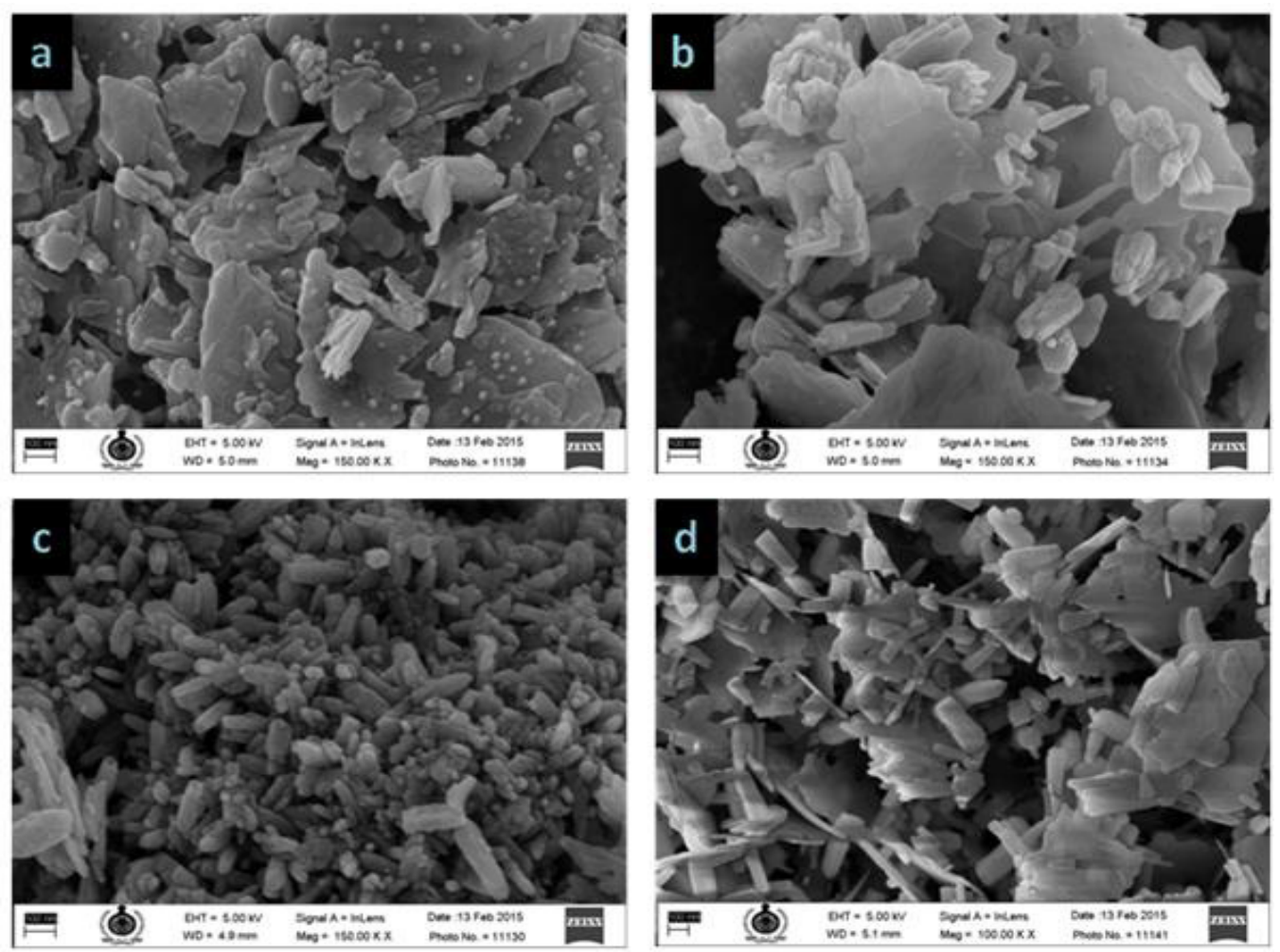

Figure 3: SEM of as-prepared a) $\mathrm{Ag} / \mathrm{ZnO} 2$; b, c) $\mathrm{Ag} / \mathrm{ZnO} 1$; d) $\mathrm{ZnO}$ (scale bar $100 \mathrm{~nm}$ ) 
Two clear silver percentages could be established with the help of EDAX analysis (Figure 4) e.g. the experiment conducted with lower concentration (40 mg) of silver salt resulted in close to 1\% (0.89\%) Ag loading on ZnO. However sample prepared with large concentration (160 mg) of $\mathrm{AgNO}_{3}$ showed about 4\% (3.89\%) of Ag loading (Table 1). It is clear from EDAX analysis that only approximately 1 and $4 \%$ loading was completed. This indicates that, ethanolic reduction is indeed slow and during the reaction time, only about $40 \%$ much reduction was possible. It is found that $\mathrm{Zn}: \mathrm{O}$ ratio is non-stoichiometric which can be because of the presence of starch thereby giving oxygen rich surfaces. It has been already described that oxygen rich $\mathrm{TiO}_{2}$ is more effective as a photocatalyst [2]. In the present case too, this may stand valid for effective photo degradation of the organic during the photocatalytic process.

\begin{tabular}{|c|c|c|c|c|c|c|}
\hline EDAX & \multicolumn{2}{|c|}{ ZnO } & \multicolumn{2}{c|}{ Ag/ZnO 1 } & \multicolumn{2}{c|}{ Ag/ZnO 2 } \\
\hline Element & Weight \% & Atomic \% & Weight \% & Atomic \% & Weight \% & Atomic \% \\
\hline O K & 42.13 & 74.84 & 34.41 & 68.31 & 34.81 & 69.09 \\
\hline Zn K & 57.87 & 25.16 & 64.70 & 31.43 & 61.27 & 29.76 \\
\hline Ag L & -- & -- & 0.89 & 0.26 & 3.92 & 1.15 \\
\hline
\end{tabular}

Table 1: Elemental composition from EDAX analysis

a)
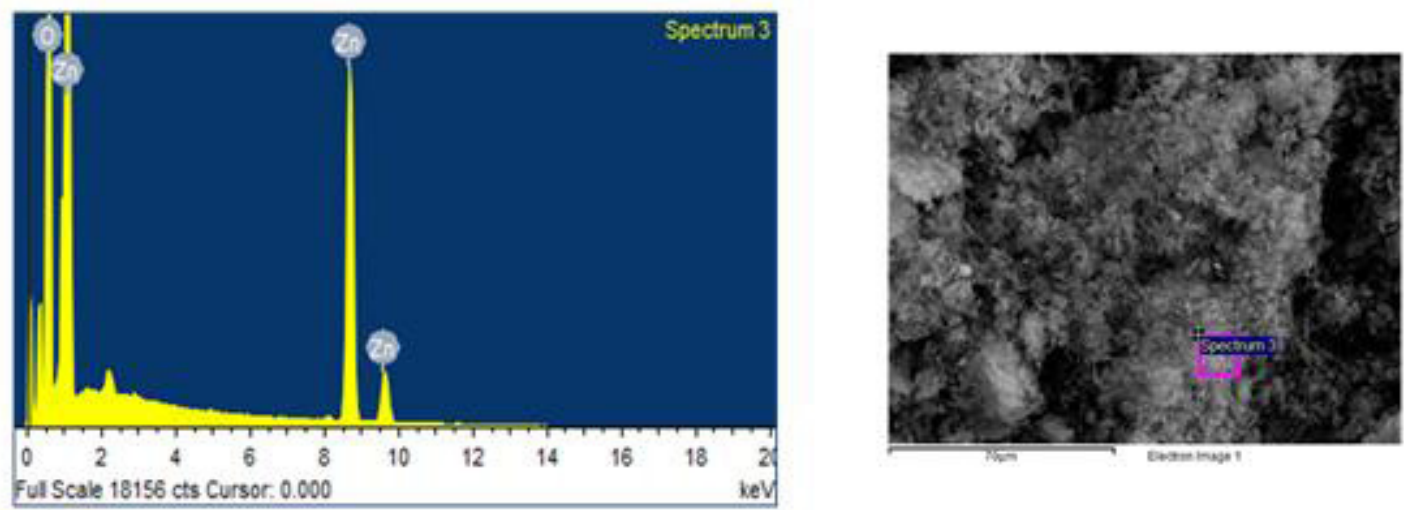

b)
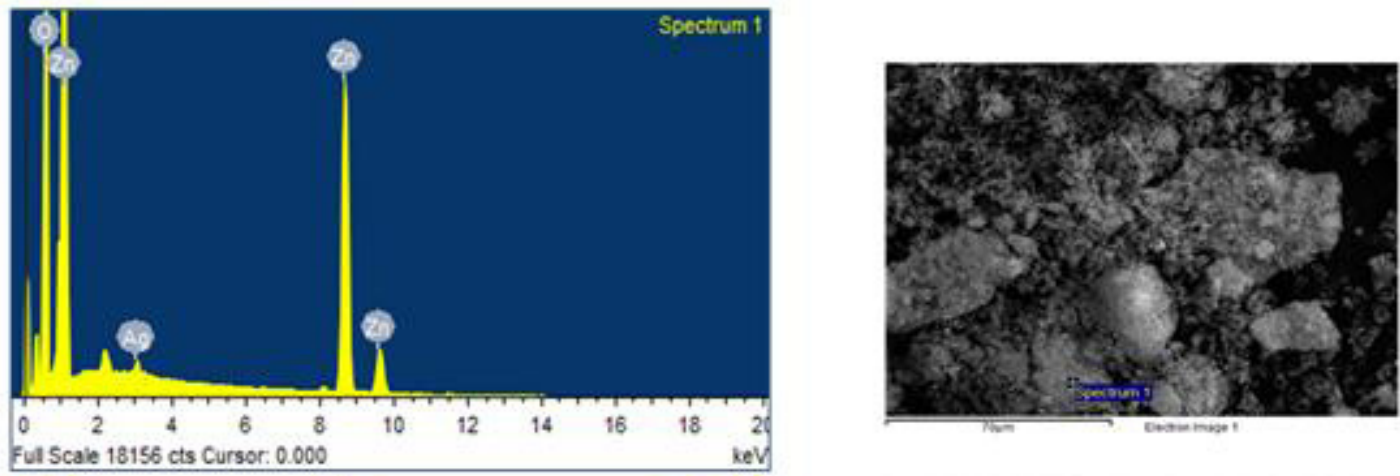

c)
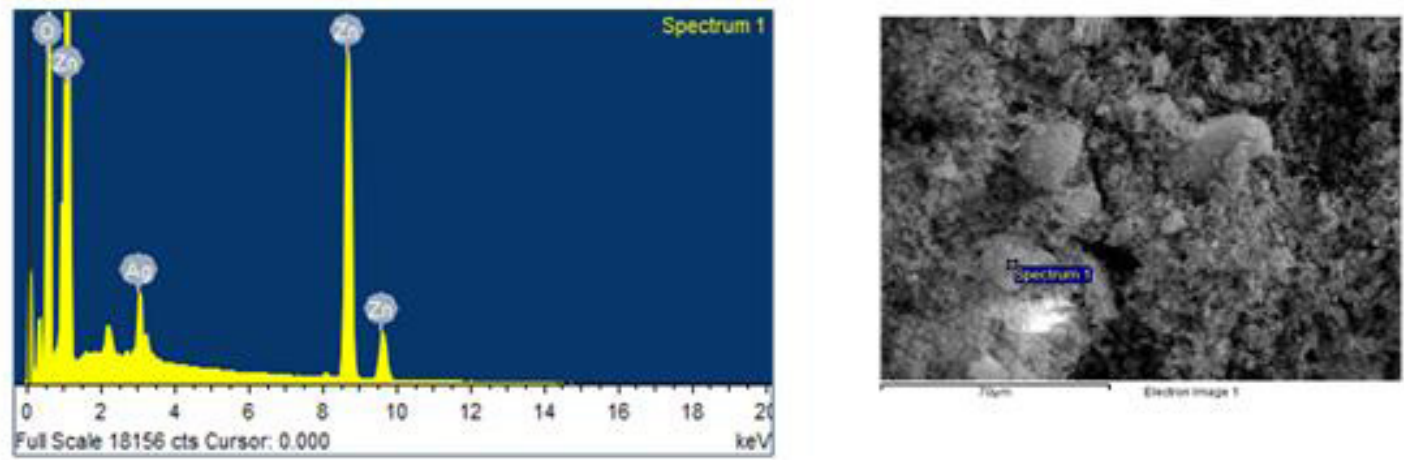

Figure 4: $\mathrm{EDAX}$ spectrum of a) $\mathrm{ZnO}$ b) $\mathrm{Ag} / \mathrm{ZnO} 1$ c) $\mathrm{Ag} / \mathrm{ZnO} 2$

\section{SEM/EDAX Analysis}

In the TEM images, the sample showed large plate and flaky appearance. The matrix plates (starch) are about 150-200 nm in length and about $20 \mathrm{~nm}$ in width. However uniformly distributed much smaller particle of $\mathrm{ZnO}$ can be considered due to presence of starch as overall matrix (Figure 5). The dark spots observed are due to homogeneously distributed silver nanoparticles on the surface of $\mathrm{ZnO}$ with the particle size of $15-20 \mathrm{~nm}$. It is seen that $\mathrm{ZnO}$ is mostly appear as rod shape which match well with morphological observations made from SEM. Due to the lack of high quality HRTEM, lattice fringes for silver and zinc oxide could not be measured to demonstrate diffusion of silver to the $\mathrm{ZnO}$ boundary. 


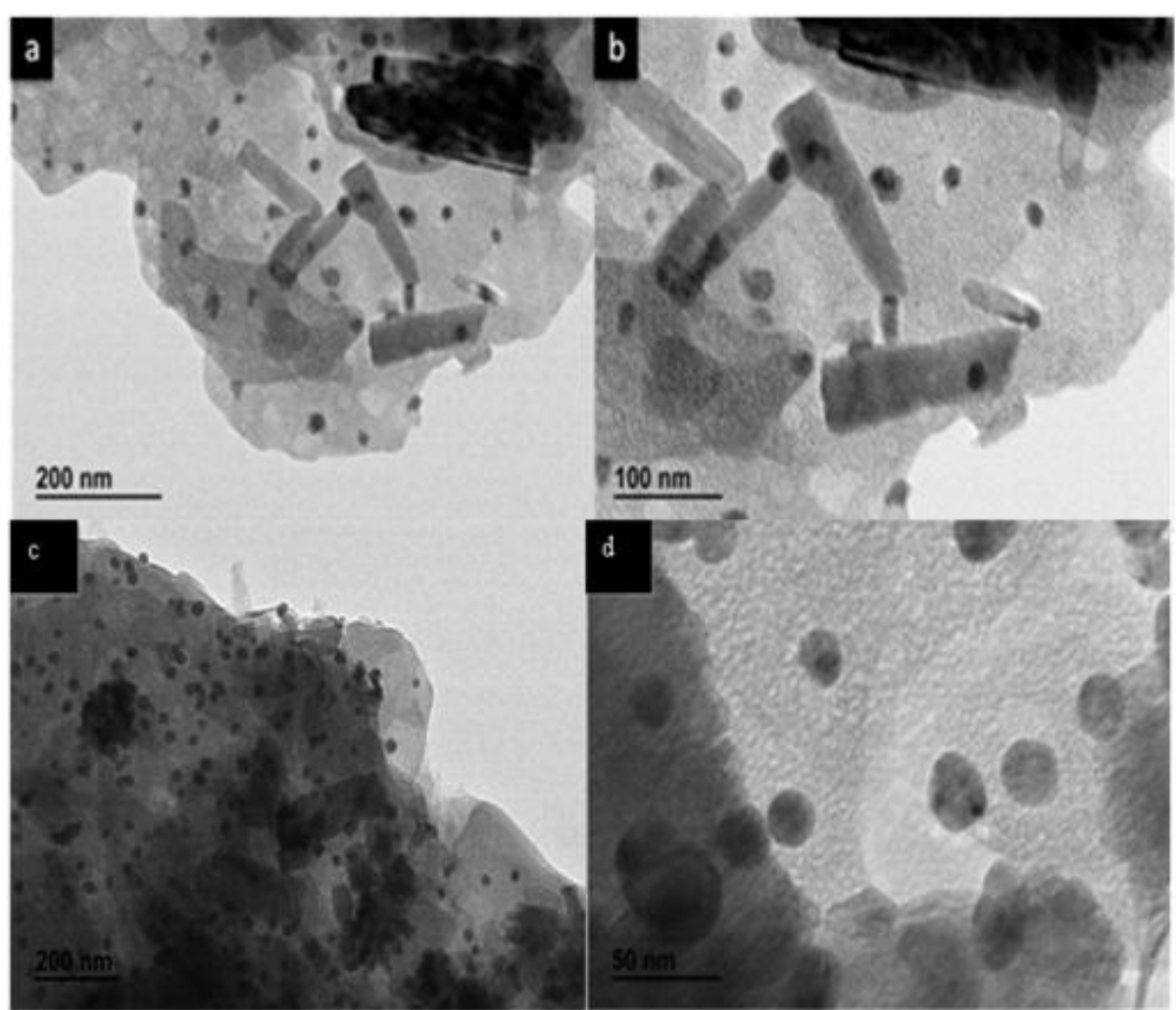

Figure 5: TEM images of as-prepared $\mathrm{Ag} / \mathrm{ZnO}(\mathrm{a}, \mathrm{b} \mathrm{Ag} / \mathrm{ZnO} 1)(\mathrm{c}, \mathrm{d} \mathrm{Ag} / \mathrm{ZnO} 2)$

\section{XPS Analysis}

In order to understand the surface components and chemical states of the $\mathrm{Ag} / \mathrm{ZnO}$ composite, XPS analysis was carried out. It could be concluded that the $\mathrm{Ag} / \mathrm{ZnO}$ consists of $\mathrm{Ag}, \mathrm{Zn}$, and $\mathrm{O}$ only and $\mathrm{Zn}$ and $\mathrm{O}$ in the case of undoped $\mathrm{ZnO}$. The carbon peak (C 1s) at 284.8 is taken as the calibration value for the curves. The peaks at $1021.4 \mathrm{eV}$ and $1044.48 \mathrm{eV}$ belongs to $\mathrm{Zn} 2 \mathrm{p}_{3 / 2}$ and $\mathrm{Zn}$ $2 \mathrm{p}_{1 / 2}$ respectively (Figure 6). The split in orbitals happens due to the formation of $\mathrm{Zn}^{2+}$ ions [21]. Slight shift to higher band energy is observed for the peak when doped with silver. The peak due to oxygen $\mathrm{O}$ (1s) can be fitted in to two symmetrical signals that of lattice oxygen and surface hydroxyl oxygen [16]. The oxygen also shows a positive shift when doped with Ag. Silver gives a characteristic peaks $367.6 \mathrm{eV}$ and 373.6. $\mathrm{eV}$ corresponding to $\mathrm{Ag} 3 \mathrm{~d}_{5 / 2}$ and $3 \mathrm{~d}_{3 / 2}$, slight deviation from bulk $\mathrm{Ag}$ value of $368.2 \mathrm{eV}$ and $374.2 \mathrm{eV}$ respectively. The $6 \mathrm{eV}$ difference between two orbitals of Ag indicates reduction of Ag metal to produce metallic silver [18]. There is a shift to lower binding energy compared to pure silver, probably due to a decrease in electron density of Ag. When $\mathrm{Ag}$ is deposited on $\mathrm{ZnO}$ surface the transfer of electrons will be from $\mathrm{Ag}$ to conduction band of $\mathrm{ZnO}$ as work function of $\mathrm{Ag}$ is smaller. Therefore an energy level is formed indicating strong interaction of $\mathrm{Ag}$ with $\mathrm{ZnO}$ particles. The positive shift of $\mathrm{Zn}$ and $\mathrm{O}$ and negative shift of silver can be due to the interaction between $\mathrm{ZnO}$ and $\mathrm{Ag}$. The binding energy of monovalent $\mathrm{Ag}$ is lower than zerovalent Ag; this must be the reason to the shift to lower energy levels for silver [39].



Figure 6: XPS patterns of $\mathrm{ZnO}$ and $\mathrm{Ag} / \mathrm{ZnO} 2 \mathrm{a}$ ) Ag 3d spectrum b)Zn (2p) Oxygen spectrum of c) $\mathrm{ZnO}$ and d)Ag/ZnO 2 


\section{UV/Vis Spectroscopy}

UV-Vis spectroscopy was performed to study the optical properties of $\mathrm{ZnO}$ nanoparticles after Ag doping. Pure ZnO particles gave rise to a peak at $373 \mathrm{~nm}$ which is similar to many reported values and is blue shifted by about $10-15 \mathrm{~nm}$ with respect to bulk value. Upon addition of the $\mathrm{Ag}$ nanoparticle to the $\mathrm{ZnO}$, a minor red shift in $\mathrm{ZnO}$ peak is noticed. In the case of sample with largest concentration of $\mathrm{Ag}$, an additional hump for silver nano-particles is observed at $429 \mathrm{~nm}$ due to localized surface Plasmon resonance (LSPR) and can indicate the morphology of silver particles close to spherical (Figure 7a). LSPR of Ag in ethanol is observed around $409 \mathrm{~nm}$ [40] and it finally settled at 429 when loaded in ZnO. This observation indicates a slight aggregation as well as increase in particle size. The Plasmon absorption peak is inversely proportional to the root of electron density of the metal [19]. Thus it is likely that LSPR will shift due to the transfer of electrons from silver to zinc oxide through $\mathrm{ZnO}-\mathrm{O}$..Ag structural arrangement in situ. Since silver has got a smaller work function than $\mathrm{ZnO}$, the transfer of electron will take place from Ag to conducting band of $\mathrm{ZnO}$ to achieve Fermi level equilibrium [37].

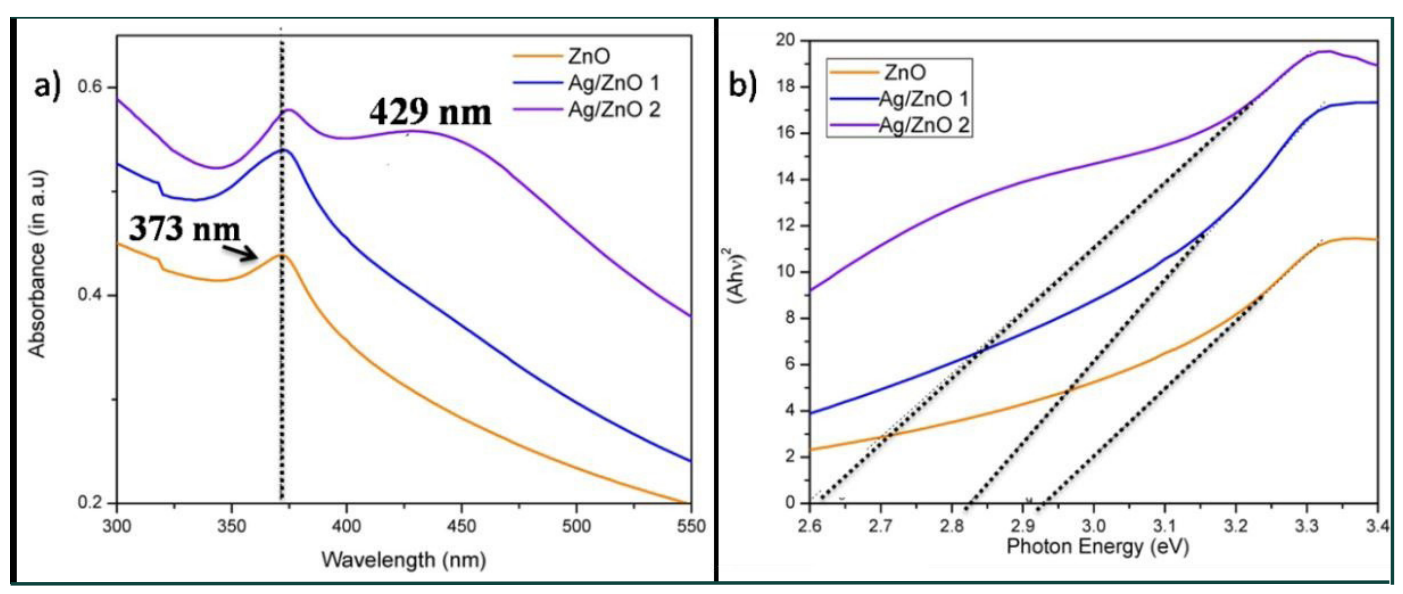

Figure 7: (a) UV/Vis Spectrum b) Tauc diagram of $\mathrm{ZnO}$ and $\mathrm{Ag} / \mathrm{ZnO}$ samples

The $h v$ values were plotted against $(a h v)^{2}$ and extended to calculate the bandgap of the nanoparticles by Tauc method [36]. The band gap (Eg) of the $\mathrm{ZnO}$ was calculated to be $2.9 \mathrm{eV}$, however for the $1 \%$ and $4 \% \mathrm{Ag}$ doped $\mathrm{ZnO}$, a reduction was observed possibly due to oxygen richness on $\mathrm{ZnO}$ surfaces. Addition of $\mathrm{Ag}$ reduced the bandgap by about $0.1-0.3 \mathrm{eV}$ to be observed at $2.8 \mathrm{eV}$ and $2.6 \mathrm{eV}$. The reduced $\mathrm{eV}$ value enabled the possibility of high photoactivity of the nanoparticles in the visible region (Figure $7 \mathrm{~b}$ ).

\section{Pholotolumiscence Spectra}

To know more about the optical properties, Pholotolumiscence data of the samples is analyzed (Figure 8). PL spectrum can provide insights on quality of crystals, structural defects (surface oxygen vacancies, Zn interstitials) and particle surfaces [39]. The room temperature PL spectrum of the samples after exciting the absorption band at $325 \mathrm{~nm}$ is recorded. The strong emission peak at 389 $\mathrm{nm}$ is the main emission along with minor emissions at 493 and $530 \mathrm{~nm}$ which can be due to bound excitons and oxygen deficiency respectively [33]. PL intensity decreased as the concentration of Ag increased. Lower photoluminescence intensity is an indicator of higher life time of electron hole pair. It shows that the adding Ag into the $\mathrm{ZnO}$ matrix inhibits the recombination rate [41].

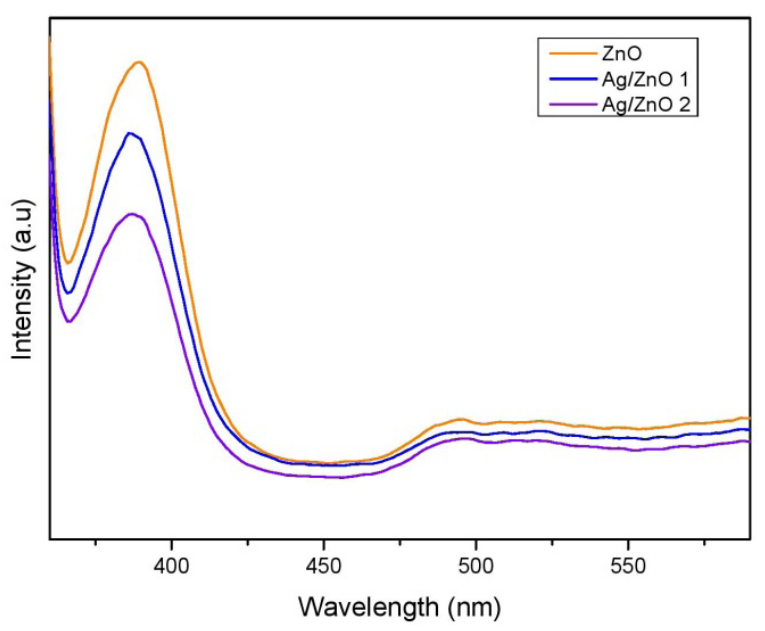

Figure 8: $\mathrm{PL}$ Spectra of $\mathrm{ZnO}$ and $\mathrm{Ag} / \mathrm{ZnO}$ samples 


\section{Photocatalytic studies}

The degradation of pollutants was done in direct sunlight at sunlight intensity of around 80000 lux $\left(\sim 590 \mathrm{~W} / \mathrm{m}^{2}\right)$. Methylene Blue is a cationic Azo dye which has been widely studied as a model dye for monitoring photocatalytic reactions. Two major absorbance peaks of methylene blue in water were located at $292 \mathrm{~nm}$ and $664 \mathrm{~nm}$, due to benzene ring and heteropolyaromatic linkage respectively. The peaks at $604 \mathrm{~nm}$ and $575 \mathrm{~nm}$ are due to the formation of dimer and trimer particles of MB in aqueous condition [43]. Chemical structure of MB is given below (Figure 9a);

a)<smiles>CN(C)c1ccc2nc3ccc(N(C)C)cc3[s+]c2c1</smiles><smiles>CN(C)c1ccc(/N=N/c2ccc(S(=O)(=O)[O-])cc2)cc1</smiles><smiles></smiles>

e)<smiles>O=S1(=O)OC(c2cc(Br)c(O)c(Br)c2)(c2cc(Br)c(O)c(Br)c2)c2ccccc21</smiles>

Figure 9: Chemical structure of dyes: a)Methylene Blue (MB) b)Rhodamine B (RhB), c)Methyl Orange (MO), d) p-nitrophenol (PNP) and e)Bromophenol Blue (BPB)

The photocatalytic degradation of Methylene Blue was evaluated with synthesized ZnO NPs and Ag/ZnO NPs under direct sunlight irradiation. The changes in the absorption intensity of MB $(664 \mathrm{~nm})$ was monitored by UV-vis spectroscopy. The photocatalytic activity of the catalyst are in the following order $\mathrm{ZnO}<\mathrm{Ag} / \mathrm{ZnO} 1(1 \mathrm{wt} \%)<\mathrm{Ag} / \mathrm{ZnO} 2(4 \mathrm{wt} \%) .1 \% \mathrm{Ag} / \mathrm{ZnO}$ improved the rate by $15 \%$, while $4 \% \mathrm{Ag} / \mathrm{ZnO}$ improved it by $40 \%$. The decolorization of $\mathrm{MB}$ ([catalyst] $=1.0 \mathrm{~g} \mathrm{~L}^{-1},[\mathrm{MB}]=0.02 \mathrm{mM}$ ) was achieved in $120 \mathrm{~min}$ in the presence of $4 \% \mathrm{Ag} / \mathrm{ZnO}$ under direct sunlight irradiation (Figure 10). This therefore confirms formulation of a solar light active $\mathrm{ZnO}$ and extremely solar light active $\mathrm{Ag} / \mathrm{ZnO}$ photocatalysit. The complete degradation of Methylene Blue has been achieved with all the samples. There are no visible signs of formation of intermediates in UV degradation curves. Rate of degradation of $664 \mathrm{~nm}$ peak is higher in the reaction thus indicating faster degradation of monomers. The MB degradation can be summarized based on the earlier reported literature as below;

$\mathrm{C}_{16} \mathrm{H}_{18} \mathrm{~N}_{3} \mathrm{~S}+24 \mathrm{O}_{2} \rightarrow 16 \mathrm{CO}_{2}+3 \mathrm{NO}_{3}{ }^{-}+\mathrm{SO}_{4}{ }^{2-}+6 \mathrm{H}++6 \mathrm{H}_{2} \mathrm{O}[44]$
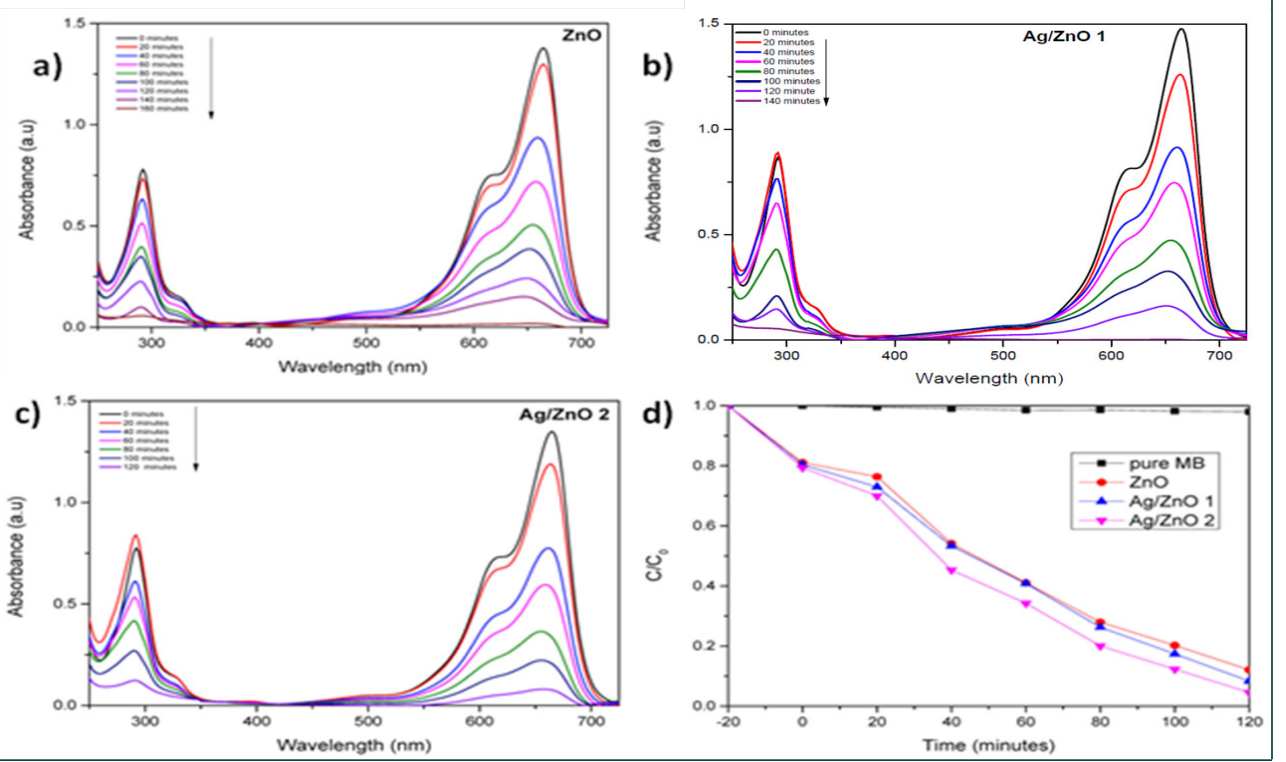

Figure 10: Degradation of $\mathrm{MB}$ with a) $\mathrm{ZnO}$, b) $1 \% \mathrm{Ag} / \mathrm{ZnO}$, c) $4 \% \mathrm{Ag} / \mathrm{ZnO}$ with $1.0 \mathrm{~g} /$ Lcatalyst concentration d) $\mathrm{C} / \mathrm{C} 0$ Vs time curves 
The amount of catalyst required is another factor which influences the photocatalytic degradation of MB. The degradation rate was faster in reactions when the concentrations of catalyst $(\mathrm{AgZnO}) 0.5 \mathrm{~g} \mathrm{~L}^{-1}$ than $0.25 \mathrm{~g} \mathrm{~L}^{-1}(4 \% \mathrm{Ag})$. In fact the solution with the higher amount of the catalyst $\left(1.0 \mathrm{~g} \mathrm{~L}^{-1}\right)$ should have offered more active surface area for the photocatalytic degradation of MB but surprisingly a decrease in the degradation rate was monitored which is presumably may be due to light scattering by excess amount of catalyst present [41] in the photocatalytic reaction system (Figure 11).
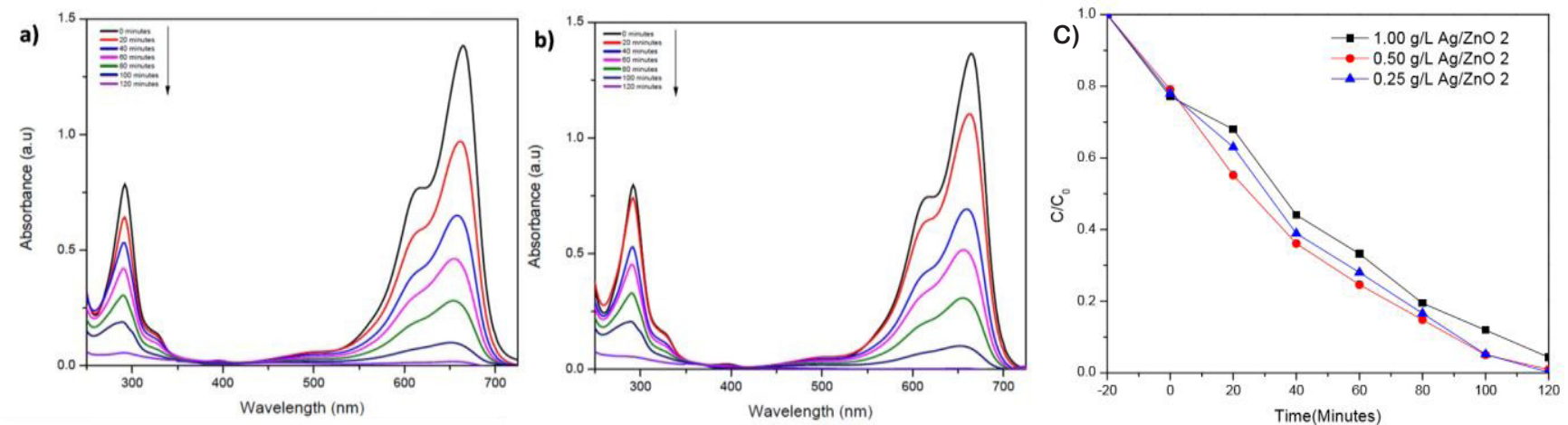

Figure 11: Degradation of MB with various concentrations of $\mathrm{Ag} / \mathrm{ZnO} 2 \mathrm{a}$ ) 0.5g/l b)0.25g/l and c) C/C0 Vs time curves for degradation of $\mathrm{MB}$ with various concentration.

In order to study the reusability of the catalyst the reaction was repeated for four cycles. The catalyst remained highly solar active and retained around $95 \%$ of its activity. This confirms that the catalyst can be effectively used in photocatalytic reactors and can be used for commercial purposes, as the leaching of $\mathrm{Ag}$ in the surface is negligible (Figure 12).

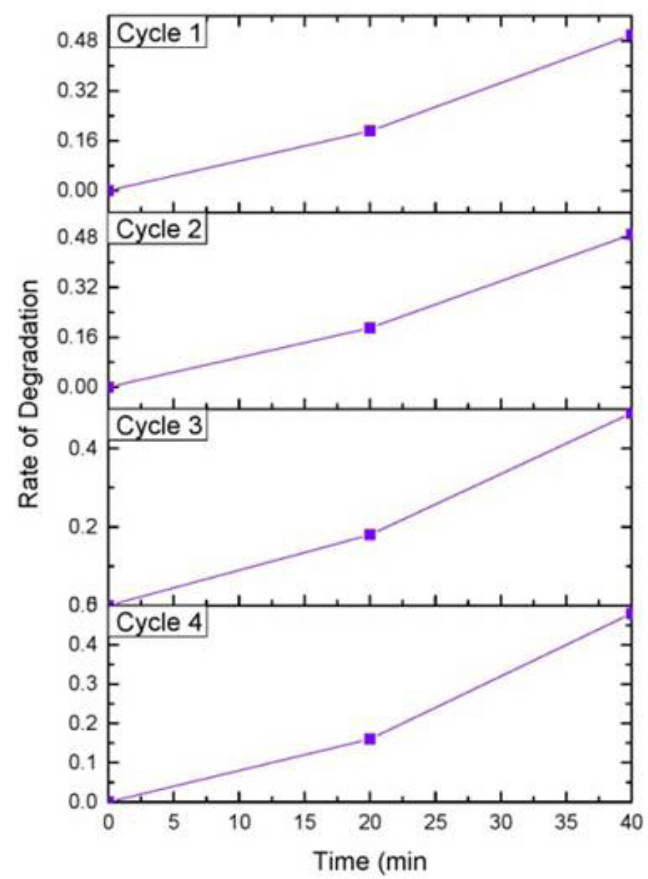

Figure 12: Recycle study of $\mathrm{Ag} / \mathrm{ZnO} 2$

Similarly, photcatalytic activities of $\mathrm{Ag} / \mathrm{ZnO}$ was tested against another industrially important dye called Rhodamine B (Figure 9b). Degradation reaction of Rhodamine $\mathrm{B}$ was quite quick in the case of $\mathrm{Ag} / \mathrm{ZnO}$, the reaction was completed in a short time of about 80 minutes; while $\mathrm{ZnO}$ took twice the time to complete the degradation process. It can be concluded that the despite synthesized $\mathrm{ZnO}$ by the current method using starch as a surfacing agent itself is quite efficient in degradation of cationic dyes, the rate of degradation can further be enhanced by addition of silver in the $\mathrm{ZnO}$ matrix (Figure $13 \mathrm{I}$ ).

Furthermore, so-generated catalyst was tested for decomposition of another industrially important dye used in textiles industry. Methyl orange (Figure 9c) is an anionic dye and can dissociate into organics under catalytic transformations. The amount of methyl orange absorbed by the catalyst is likely to be less due to presence of oxygen rich surfaces because of the negative charges on the catalyst surface [13]. Indeed, when $\mathrm{ZnO}$ alone was employed as a catalyst the degradation was poorer (only 90\% in 240 min) which however improved in presence of silver in $\mathrm{ZnO}$ confirming that presence of silver create scope for anionic dye to effectivel- 
$y$ absorb on the catalyst surface. Thus the complete reaction takes place (almost 100\%) in 160 minutes as against 240 minutes of radiation with $\mathrm{ZnO}$ alone. The performance of plane $\mathrm{ZnO}$ in our case was still better than many reported articles thus further confirming that starch passivating ZnO surfaces are effective by themselves (Figure 13 II). Similarly, when the two catalysts studied in this work, were tested for degradation of Bromophenol Blue (Figure 9e), another anionic dye, the reaction rate was fast for Ag/ $\mathrm{ZnO}$ than $\mathrm{ZnO}$ alone (Figure $14 \mathrm{I}$ ).

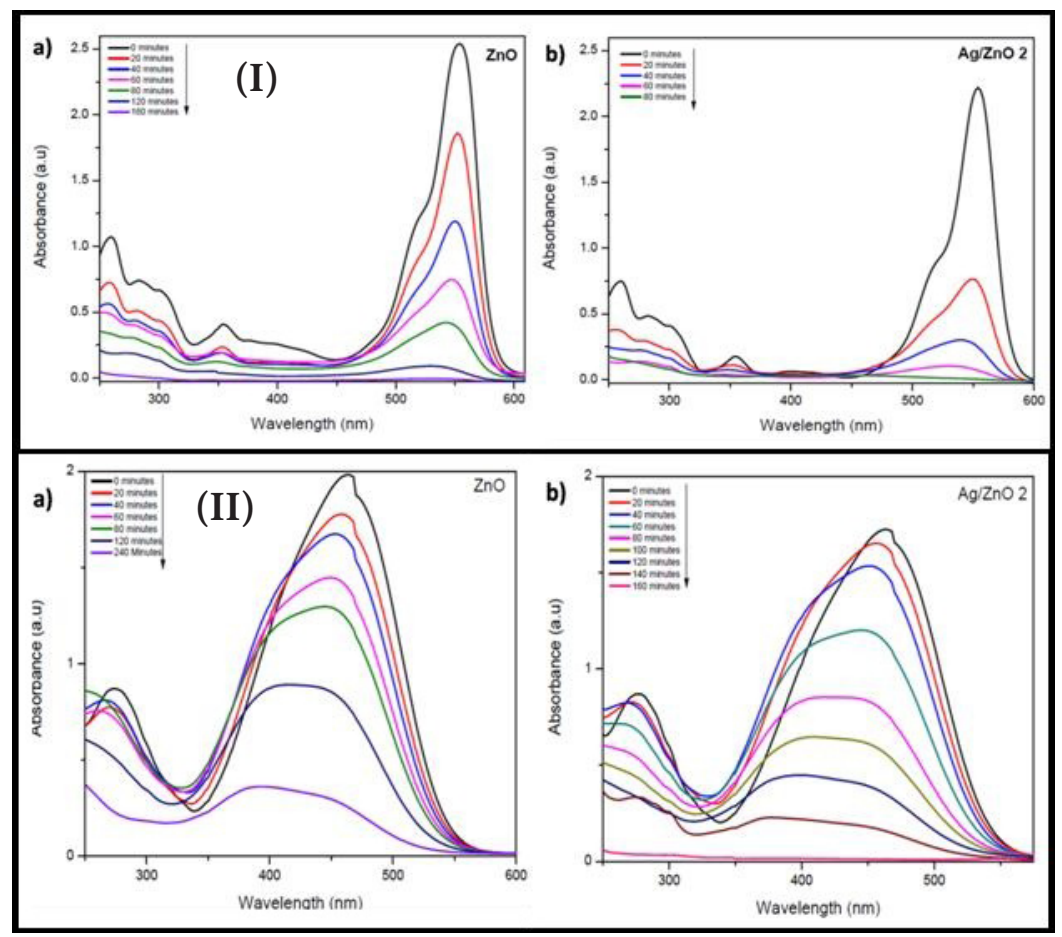

Figure 13: Photodegradation of (I) Rhodamine $\mathrm{B}$, (II) $\mathrm{MO}$ using a) bare $\mathrm{ZnO}$ and b) $\mathrm{Ag} / \mathrm{ZnO} 2$
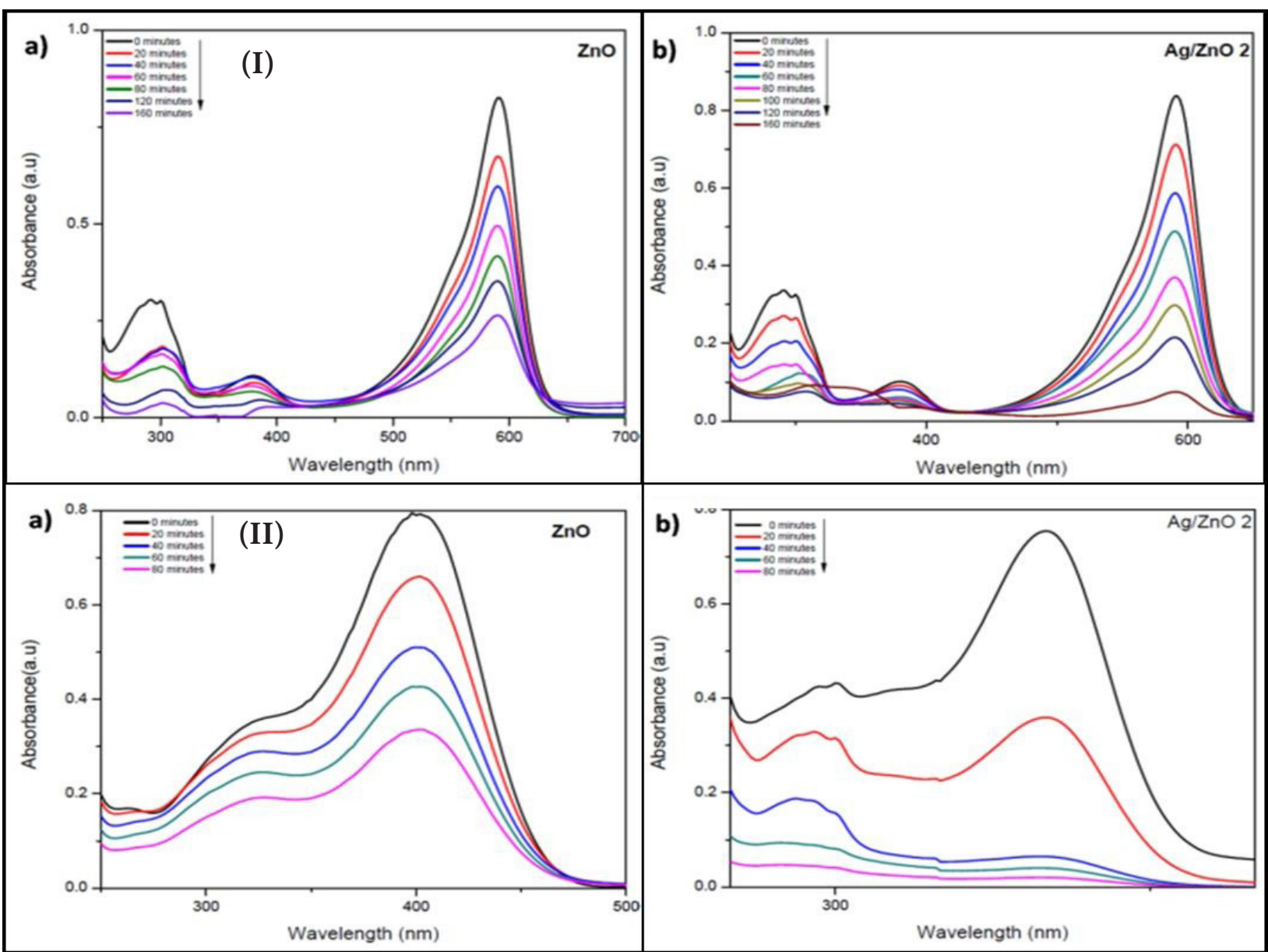

Figure 14: Degradation of (I) Bromophenol Blue, (II) p-nitrophenol using a) $\mathrm{ZnO}$ and b) $\mathrm{Ag} / \mathrm{ZnO} 2$ 
Additionally, it is important in totality to understand need of effluent treatment. There is large number of organic pollutants in industrial effluent and providing a solution for total degradation of the contaminants, will obviously be a preferred technology. In order to establish the scope of current catalyst to have potential as a total solution, present article studies on phenol and phenolic compounds also which are common contaminants in industrial waste water. The degradation of phenols is challenging due to its stability and water solubility. For p-nitrophenol (Figure 9d) the reaction rates were very less for bare $\mathrm{ZnO}$, the reaction was quite faster for $\mathrm{Ag} / \mathrm{ZnO}$. An intermediate peak, probably due to hydroquinone formation $[45,46]$ is visible in the reaction for $\mathrm{Ag} / \mathrm{ZnO}$ (Figure 14 II). By showing the efficiency of degradation for decomposition of five types of water pollutants, (Table 2) it is proposed that such type of catalyst can offer solution to water pollution problem that is a global concern.

\begin{tabular}{|c|c|c|c|c|}
\hline \multirow{2}{*}{ Pollutants } & $\begin{array}{c}\text { Concentration of pollutants } \\
(\mathbf{m M})\end{array}$ & $\begin{array}{c}\text { Time for degradation } \\
(\mathbf{m i n})\end{array}$ & \multicolumn{2}{|c|}{ Degradation (\%) by } \\
\cline { 3 - 5 } & 0.02 & 120 & $\mathbf{Z n O}$ & $\mathbf{A g} / \mathbf{Z n O} 2$ \\
\hline Methylene Blue (MB) & 0.02 & 80 & $80 \%$ & $98 \%$ \\
\hline Rhodamine B(RhB) & 0.01 & 160 & $73 \%$ & $99.9 \%$ \\
\hline Methyl Orange(MO) & 0.005 & 160 & $64 \%$ & $92 \%$ \\
\hline Bromophenol Blue (BPB) & 0.01 & 80 & $70 \%$ & $96 \%$ \\
\hline p-nitrophenol (PNP) & & & & \\
\hline
\end{tabular}

Table 2: Efficiency of photocatalysts

\section{Photocatalytic Mechanism}

In the present study, pure ZnO NPs showed good photocatalytic activity which is believed to be due to two factors, band gap narrowing due to high oxygen concentration in the base material and due to excitation of the absorbed pollutant because of dye sensitization. XPS and EDAX data clearly show excess oxygen in the catalysts sample; probably due to the oxidative action of starch. Generally, Oxygen vacancies in the $\mathrm{ZnO}$ crystal can act as recombination centres, thereby decreasing the photocatalytic efficiency. Lower recombination rates can be the reasons for higher efficiency [2]. PL curve of ZnO shows possibility of low recombination rates and almost negligible impurity peaks. Chemisorbed dyes produce single and triple excited states when excited by visible light. Electrons are transferred to the conduction band of semiconductor from the excited dyes. In the process hydroxyl radicals are generated which carry out the degradation reaction forward [18].

When $\mathrm{ZnO}$ is doped with Ag, nano hetero junctions are formed. Optimum loading of Ag, as SEM and TEM data indicates, seems to be the reason for better photocatalytic property of $4 \% \mathrm{Ag} / \mathrm{ZnO}(\mathrm{Ag} / \mathrm{ZnO} 2)$ than $1 \% \mathrm{Ag} / \mathrm{ZnO}$ (Ag/ZnO 1). The electronic interaction in the metal semiconductor junction suppresses the recombination of the charge carriers. The current mechanism can be considered as similar to that has been reported by other researchers [42] where they have reported that due to localized surface plasmon resonance (LSPR) process, Ag particles act like an antenna for trapping sunlight radiation. LSPR acts as a locally excited electric field and the electrons in the valence band of excited silver nanoparticles ( $\mathrm{Ag}^{*}$ in reaction 6$)$ get transferred to the conduction band of $\mathrm{ZnO}$ and showing formation of $\mathrm{ZnO}(\mathrm{e})$ [5]. Thereafter, $\mathrm{ZnO}$ in $\mathrm{Ag} / \mathrm{ZnO}$ will transfer the charge to Ag NPs, thus increasing the rate of separation of photogenerated electron hole pairs. Holes in $\mathrm{ZnO}$ react with water leading to the formation of hydroxyl radicals and electrons in conduction band of Ag nanoparticle produce superoxide $\left(\mathrm{O}_{2} \cdot{ }^{-}\right)$radicals. These radical mineralize the pollutant molecules to basic substances $[42,47]$. The overall pictorial photocatalytic mechanism of degradation of $\mathrm{MB}$ in presence of $\mathrm{Ag} / \mathrm{ZnO}$ under sunlight is shown in figure 15 [41]. The following reaction chain is the normal process [19,48];

$$
\begin{aligned}
& \mathrm{Ag}+\mathrm{hv} \rightarrow \mathrm{Ag}^{*} \\
& \mathrm{Ag}^{*}+\mathrm{ZnO} \rightarrow \mathrm{Ag}^{\bullet+}+\mathrm{ZnO}(\mathrm{e}) \\
& \mathrm{ZnO}(\mathrm{e})+\mathrm{O}_{2} \rightarrow \mathrm{ZnO}+\mathrm{O}_{2} \bullet^{-} \\
& \mathrm{O}_{2} \bullet^{-}+\mathrm{H}+\rightarrow \bullet \mathrm{OOH} \\
& \bullet \mathrm{OOH}+\mathrm{ZnO}(\mathrm{e})+\mathrm{H}^{+} \rightarrow \mathrm{H}_{2} \mathrm{O}_{2}+\mathrm{ZnO} \\
& \mathrm{H}_{2} \mathrm{O}_{2}+\mathrm{ZnO}(\mathrm{e}) \rightarrow \bullet \mathrm{OH}+\mathrm{OH}^{-}+\mathrm{ZnO}
\end{aligned}
$$

Organic Pollutants $+\bullet \mathrm{OH}($ or $\bullet \mathrm{OOH}) \rightarrow$ Degraded Products

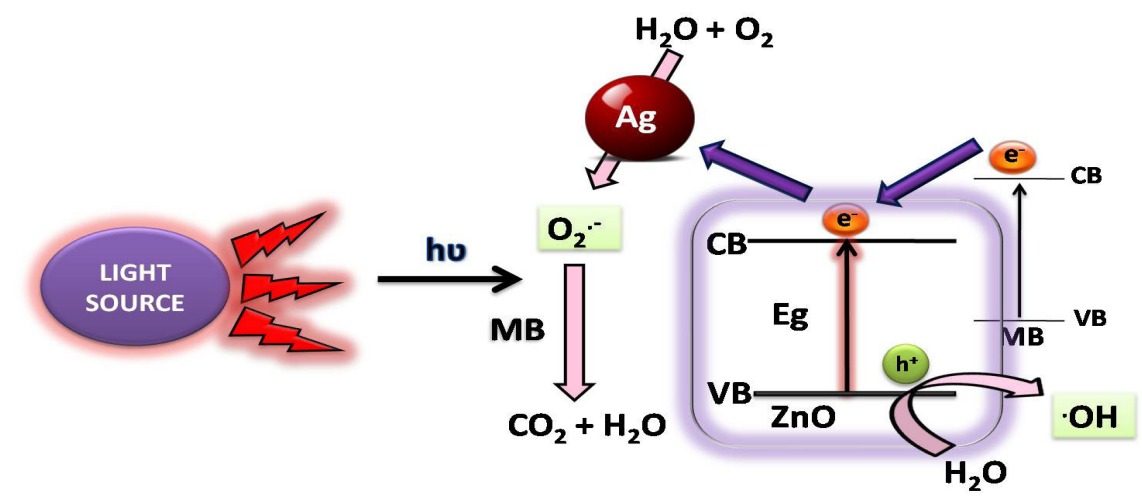

Figure 15: Proposed mechanism for degradation of $\mathrm{MB}$ via electronic events in presence of $\mathrm{Ag} / \mathrm{ZnO}$ catalyst under sunlight 
Additionally, in case of plasmonic photocatalyst $(\mathrm{Ag} / \mathrm{ZnO})$, intensive local electric field LSPR may create excitation of more electrons and holes and heat up the surrounding environment to increase the reaction rate and the mass transfer thereby polarizing the nonpolar molecules for better adsorption and absorption of pollutants with $\mathrm{Ag} / \mathrm{ZnO}$ [30]. This local electric field may also have factored in better performance in the present study.

\section{Conclusion}

$\mathrm{Ag} / \mathrm{ZnO}$ photocatalyst which is highly efficient under solar radiation has been prepared using starch. The catalyst was characterized and showed good crystalline properties and showed excessive surface oxygen which may be crucial to the overall catalytic activity during the testing and evaluation of its photocatalytic performance. Presence of silver in the zinc oxide nanostructure enhances the band-gap towards visible region to convert ultraviolet active zinc oxide to a visible light catalyst for its evaluation under direct sunlight. It is observed that presence of silver enhances the photcatalysis and also improves properties like absorption of dyes on the surface. The comparative study of the photocatalytic activity revealed following order for catalyst $\mathrm{ZnO}<\mathrm{Ag} / \mathrm{ZnO} 1(1 \mathrm{wt} \%)<$ $\mathrm{Ag} / \mathrm{ZnO} 2$ (4 wt \%). 1\% Ag/ZnO improved the rate by 15\%, while 4\% $\mathrm{Ag} / \mathrm{ZnO}$ improved it by $40 \%$. The highest efficiency (99.9\%) was obtained for $\mathrm{Ag} / \mathrm{ZnO} 2$ photocatalyst against rhodamine B. The so-generated catalysts were able to degrade both cationic (methylene blue, rhodamine B) and anionic dyes (methyl orange, bromophenol blue and phenols ( $\mathrm{p}$ - nitrophenol). It retained its activity even after third cycle. The catalyst can be used as a directly under the sunlight thereby enhancing the scope for industrial application.

\section{Acknowledgements}

This work was supported by DIAT (DU). Special Thanks to Dr K R Patil, Centre for Material Characterization, National Chemical Laboratories, Pune for XPS facility, Mr Ujjawal K Bhagat for experimental assistance in synthesis of $\mathrm{ZnO}$.

\section{References}

1. Fujishima A, Honda K (1972) Electrochemical Photolysis of Water at a Semiconductor Electrode. Nature 238: 37-38.

2. a) Etacheri V, Seery MK, Hinder SJ, Pillai SC (2011) Oxygen Rich Titania: A Dopant Free, High Temperature Stable, and Visible Light Active Anatase Photocatalyst. Adv Funct Materials 21: 3744-52.

b) Liu S, Tang ZR, Sun Y, Colmenares JC, Xu YJ (2015) One-dimension-based spatially ordered architectures for solar energy conversion. Chem Soc Rev 44: 5053-75.

3. Jang YJ, Simer C, Ohm T (2006) Comparison of zinc oxide nanoparticles and its nano-crystalline particles on the photocatalytic degradation of methylene blue. Mater Res Bull 41: 67-77.

4. a) Li S, Ma Z, Zhang J, Wu Y, Gong Y (2008) A comparative study of photocatalytic degradation of phenol of $\mathrm{TiO}_{2}$ and $\mathrm{ZnO}$ in the presence of manganese dioxides. Catal Today 139: 109-112.

b) Zhang N, Yang MQ, Liu S, Sun Y, Xu YJ (2015) Waltzing with the Versatile Platform of Graphene to Synthesize Composite Photocatalysts. Chem Rev 115: 10307-77.

5. Zhou X, Liu G, Yu J, Fan W (2012) Surface plasmon resonance-mediated photocatalysis by noble metal-based composites under visible light. J Mater Chem 22: 21337-54.

6. Primo A, Corma A, García H (2011) Titania supported gold nanoparticles as photocatalyst. Phys Chem Chem Phys 13: 886-910.

7. Collado L, Jana P, Sierra B, Coronado J, Pizarro P, et al. (2013) Enhancement of hydrocarbon production via artificial photosynthesis due to synergetic effect of Ag supported on $\mathrm{TiO}_{2}$ and $\mathrm{ZnO}$ semiconductors. Chem Eng J 224: 128-35.

8. Cho IS, Chen Z, Forman AJ, Kim DR, Rao PM, et al. (2011) Branched $\mathrm{TiO}_{2}$ nanorods for photoelectrochemical hydrogen production. Nano lett 11: 4978-84.

9. Ravishankar T, Manjunatha K, Ramakrishnappa T, Nagaraju G, Kumar D, et al. (2014) Comparison of the photocatalytic degradation of trypan blue by undoped and silver-doped zinc oxide nanoparticles. Mater Sci Semicon Proc 26: 7-17.

10. Jasso-Salcedo AB, Palestino G, Escobar-Barrios VA (2014) Effect of Ag, pH, and time on the preparation of Ag-functionalized zinc oxide nanoagglomerates as photocatalysts. J Catal 318: 170-8.

11. Wang Y, Wang Q, Zhan X, Wang, Safdar M, et al. (2013) Visible light driven type II heterostructures and their enhanced photocatalysis properties: a review. Nanoscale 5: 8326-39.

12. Height MJ, Pratsinis SE, Mekasuwandumrong O, Praserthdam P (2006) Ag-ZnO catalysts for UV-photodegradation of methylene blue. Appl Catal B-Environ 63: 305-12.

13. Rauf M, Ashraf SS (2009) Fundamental principles and application of heterogeneous photocatalytic degradation of dyes in solution. Chem Eng J 151: 10-8.

14. Houas A, Lachheb H, Ksibi M, Elaloui E, Guillard C, et al. (2001) Photocatalytic degradation pathway of methylene blue in water. Appl Catal B-Environ 31: $145-57$.

15. Rehman S, Ullah R, Butt A, Gohar N (2009) Strategies of making $\mathrm{TiO}_{2}$ and $\mathrm{ZnO}$ visible light active. J Hazard Mater 170: 560-9.

16. Wang J, Wang Z, Huang B, Ma Y, Liu Y, et al. (2012) Oxygen vacancy induced band-gap narrowing and enhanced visible light photocatalytic activity of ZnO. ACS Appl Mater Interfaces 4: 4024-30.

17. Zheng Y, Zheng L, Zhan Y, Lin X, Zheng Q, et al. (2007) Ag/ZnO heterostructure nanocrystals: synthesis, characterization, and photocatalysis. Inorg Chem 46: 6980-6.

18. Deng Q, Duan X, Ng DH, Tang H, Yang Y, et al. (2012) Ag nanoparticle decorated nanoporous ZnO microrods and their enhanced photocatalytic activities. ACS Appl Mater Interfaces 4: 6030-7.

19. Han Z, Ren L, Cui Z, Chen C, Pan H, et al. (2012) Ag/ZnO flower heterostructures as a visible-light driven photocatalyst via surface plasmon resonance. Appl Catal B-Environ 126: 298-305.

20. Li Y, Zhao X, Fan W (2011) Structural, electronic, and optical properties of Ag-doped ZnO nanowires: first principles study. J Phys Chem C 115: $3552-7$. 
21. Hong Y, Tian C, Jiang B, Wu A, Zhang Q, et al. (2013) Facile synthesis of sheet-like ZnO assembly composed of small ZnO particles for highly efficient photocatalysis. J Mater Chem A 1: 5700-8.

22.a) Zhang G, Shen X, Yang Y (2011) Facile synthesis of monodisperse porous ZnO spheres by a soluble starch-assisted method and their photocatalytic activity.

J Phys Chem C 115: 7145-52.

b) Khanna PK, Singh N, Kulkarni D, Deshmukh S, Charan S, et al. (2007) Water based simple synthesis of re-dispersible silver nano-particles. Materials Letters 61: 3366-70.

c) Khanna PK, Bhagat UK, More PV (2015) Study of Zinc Oxide Nanofluids for Heat Transfer Application. SAJ Nanosci Nanotech 1: 101.

d) Khanna PK, Singh N, Charan S (2007) Synthesis of nano-particles of anatase $\mathrm{TiO}_{2}$ and preparation of its optically transparent film in PVA. Materials Letters 61: 4725-30.

23. Raveendran P, Fu J, Wallen SL (2003) completely "green" synthesis and stabilization of metal nanoparticles. J Am Chem Soc 125: 13940-1.

24. Lin ST, Thirumavalavan M, Jiang TY, Lee JF (2014)Synthesis of ZnO/Zn nano photocatalyst using modified polysaccharides for photodegradation of dyes. Carbohyd Polym 105: 1-9.

25.a) Zak AK, Majid WA, Mahmoudian M, Darroudi M, Yousefi R (2013) Starch-stabilized synthesis of ZnO nanopowders at low temperature and optical properties study. Adv Pow Technol 24: 618-24.

b) Ye C, Bando Y, Shen G, Golberg D (2006) Thickness-dependent photocatalytic performance of ZnO nanoplatelets. J Phys Chem B 110: $15146-151$.

c) Makarova OV, Rajh T, Thurnauer MC, Martin A, Kemme PA, et al. (2000) Surface modification of $\mathrm{TiO}_{2}$ nanoparticles for photochemical reduction of nitrobenzene. Environ Sci Technol 34: 4797-803.

26.a) Xie S, Lu X, Zhai T, Li W, Yu M, et al. (2012) Enhanced photoactivity and stability of carbon and nitrogen co-treated ZnO nanorod arrays for photoelectrochemical water splitting. J Mater Chem 22: 14272-5.

b) Han C, Yang MQ, Weng B, Xu YJ (2014) Improving the photocatalytic activity and anti-photocorrosion of semiconductor ZnO by coupling with versatile carbon. Phys Chem Chem Phys 16: 16891-903.

c) Weng B, Yang MQ, Zhang N, Xu YJ (2014) Toward the enhanced photoactivity and photostability of ZnO nanospheres via intimate surface coating with reduced graphene oxide. J Mater Chem A 2: 9380-9.

27. Ahmad M, Ahmed E, Zhang Y, Khalid N, Xu J, et al. (2013) Preparation of highly efficient Al-doped ZnO photocatalyst by combustion synthesis. Curr Appl Phys 13: 697-704.

28. Liu S, Li C, Yu J, Xiang Q (2011) Improved visible-light photocatalytic activity of porous carbon self-doped ZnO nanosheet-assembled flowers. Cryst Eng Comm 13: 2533-41.

29. Vignesh K, Suganthi A, Rajarajan M, Sara S (2012) Photocatalytic activity of AgI sensitized ZnO nanoparticles under visible light irradiation. Powd Technol 224: $331-7$.

30. Zhang X, Chen YL, Liu R-S, Tsai DP (2013) Plasmonic photocatalysis. Rep Prog Phys 76: 046401.

31. Awazu K, Fujimaki M, Rockstuhl C, Tominaga J, Murakami H, et al. (2008) A plasmonic photocatalyst consisting of silver nanoparticles embedded in titanium dioxide. J Am Chem Soc 130: 1676-80.

32. Dao TD, Han G, Arai N, Nabatame T, Wada Y, et al. (2015) Plasmon-mediated photocatalytic activity of wet-chemically prepared ZnO nanowire arrays. Phys Chemi Chem Phys 17: 7395-403.

33. Georgekutty R, Seery MK, Pillai SC (2008) A highly efficient Ag-ZnO photocatalyst: synthesis, properties, and mechanism. J Phys Chem C 112: 13563-70.

34. Mondal C, Pal J, Ganguly M, Sinha AK, Jana J, et al. (2014) A one pot synthesis of Au-ZnO nanocomposites for plasmon-enhanced sunlight driven photocatalytic activity. New J Chem 38: 2999-3005.

35. Liu Y, Wei S, Gao W (2015) Ag/ZnO heterostructures and their photocatalytic activity under visible light: Effect of reducing medium. J Hazard Mater 287: 59-68. 36. Yildırım OA, Unalan HE, Durucan C (2013) Highly Efficient Room Temperature Synthesis of Silver-Doped Zinc Oxide (ZnO:Ag) Nanoparticles: Structural, Optical, and Photocatalytic Properties. J Am Ceram Soc 96: 766-73.

37.a) Ansari SA, Khan MM, Ansari MO, Lee J, Cho MH (2013) Biogenic synthesis, photocatalytic, and photoelectrochemical performance of Ag-ZnO nanocomposite. J Phys Chem C 117: 27023-30,

b) Pan X, Yang MQ, Fu X, Zhang N, Xu Y-J (2013) Defective $\mathrm{TiO}_{2}$ with oxygen vacancies: synthesis, properties and photocatalytic applications. Nanoscale 5: 3601-14.

c) X Pan, Yang MQ, Xu YJ (2014) Morphology control, defect engineering and photoactivity tuning of ZnO crystals by graphene oxide - a unique 2D macromolecular surfactant. Phys Chem Chem Phys 16: 5589-99.

38. Vayssieres L (2003) Growth of arrayed nanorods and nanowires of $\mathrm{ZnO}$ from aqueous solutions. Adv Mater 15: 464-6.

39. Sun F, Qiao X, Tan F, Wang W, Qiu X (2012) One-step microwave synthesis of Ag/ZnO nanocomposites with enhanced photocatalytic performance. J Mater Sci 47: 7262-8.

40. Pal A, Shah S, Devi S (2009) Microwave-assisted synthesis of silver nanoparticles using ethanol as a reducing agent. Mater Chem Phys 114: 530-2.

41. Kaviya S, Prasad E (2015) Biogenic synthesis of ZnO-Ag nano custard apples for efficient photocatalytic degradation of methylene blue by sunlight irradiation. RSC Adv 5: 17179-185.

42. Liu X, Li W, Chen N, Xing X, Dong C, et al. (2015) Ag-ZnO heterostructure nanoparticles with plasmon-enhanced catalytic degradation for Congo red under visible light. RSC Adv 5: 34456-65.

43. Heger D, Jirkovsky J, Klan P (2005) Aggregation of methylene blue in frozen aqueous solutions studied by absorption spectroscopy. J Phys Chem A 109: 6702-9. 44. Lachheb H, Puzenat E, Houas A, Ksibi M, Elaloui E, et al. (2002) Photocatalytic degradation of various types of dyes (Alizarin S, Crocein Orange G, Methyl Red, Congo Red, Methylene Blue) in water by UV-irradiated titania. Appl Catal B-Environ 39: 75-90.

45. Maurino V, Minero C, Pelizzetti E, Piccinini P, Serpone N, et al. (1997) The fate of organic nitrogen under photocatalytic conditions: degradation of nitrophenols and aminophenols on irradiated $\mathrm{TiO}_{2}$. J Photochem Photobiol A 109: 171-6.

46. Nagaveni K, Sivalingam G, Hegde M, Madras G (2004) Photocatalytic degradation of organic compounds over combustion-synthesized nano- $\mathrm{TiO}_{2}$. Environ Sci Technol 38: 1600-4.

47. Xiao M, Jiang R, Wang F, Fang C, Wang J, et al. (2013) Plasmon-enhanced chemical reactions. J Mater Chem A 1: 5790-805.

48. Kochuveedu ST, Jang YH, Kim DH (2013) A study on the mechanism for the interaction of light with noble metal-metal oxide semiconductor nanostructures for various photophysical applications. Chem Soc Rev 42: 8467-93. 


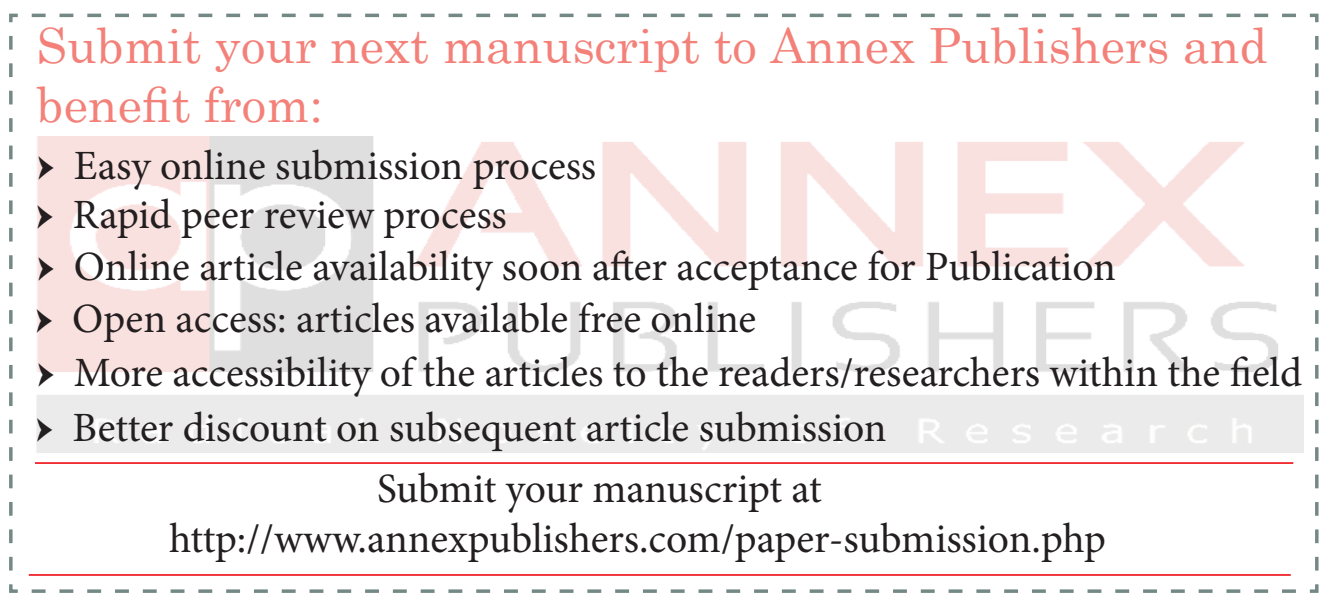

